\title{
Quasi parton distributions and the gradient flow
}

\author{
Christopher Monahan ${ }^{a}$ and Kostas Orginos ${ }^{b, c}$ \\ ${ }^{a}$ New High Energy Theory Center and Department of Physics and Astronomy, \\ Rutgers, the State University of New Jersey, \\ 136 Frelinghuysen Road, Piscataway, NJ 08854-8019, U.S.A. \\ ${ }^{b}$ Physics Department, College of William and Mary, \\ Williamsburg, Virginia 2318\%, U.S.A. \\ ${ }^{c}$ Thomas Jefferson National Accelerator Facility, \\ Newport News, Virginia 23606, U.S.A. \\ E-mail: chris.monahan@rutgers.edu, kostas@wm.edu
}

ABSTRACT: We propose a new approach to determining quasi parton distribution functions (PDFs) from lattice quantum chromodynamics. By incorporating the gradient flow, this method guarantees that the lattice quasi PDFs are finite in the continuum limit and evades the thorny, and as yet unresolved, issue of the renormalization of quasi PDFs on the lattice. In the limit that the flow time is much smaller than the length scale set by the nucleon momentum, the moments of the smeared quasi PDF are proportional to those of the lightfront PDF. We use this relation to derive evolution equations for the matching kernel that relates the smeared quasi PDF and the light-front PDF.

KEYWorDs: Lattice QCD, Lattice Quantum Field Theory

ARXIV EPRINT: 1612.01584 


\section{Contents}

1 Introduction 1

2 Distribution functions 3

2.1 Bare PDFs 3

2.2 Renormalized PDFs 4

2.3 Smeared quasi PDFs 5

3 Relation to light-front distributions $\quad 6$

3.1 Short-distance expansion 8

4 DGLAP-like equation for the matching kernel 10

$\begin{array}{llr}5 & \text { Conclusion } & 11\end{array}$

\section{Introduction}

Quantum chromodynamics (QCD) is the theory of the strong nuclear force that connects hadronic bound states to their partonic constituents, quarks and gluons. Although quarks and gluons cannot be directly accessed in experiments, the connection between hadrons and partons can be characterized through parton distribution functions (PDFs).

PDFs capture aspects of hadron structure associated with the momentum, angular momentum and spin of the constituent quarks and gluons, and play a central role in our understanding of high energy hadronic scattering processes (see, for example, [1-3]). Through factorization, the scattering amplitudes of simple scattering processes, such as deep inelastic scattering and Drell-Yan production, can be expressed as the convolution of perturbative coefficients and PDFs, which encapsulate the nonperturbative dynamics of QCD at hadronic scales.

In principle, the direct calculation of PDFs from QCD will provide new insight into hadronic structure, more stringent tests of QCD, and reduced systematic uncertainties in high energy scattering experiments. At present, however, the only systematic method for ab initio, nonperturbative QCD calculations is lattice QCD, in which QCD is formulated on a discrete Euclidean hypercube. PDFs are defined in terms of matrix elements of lightfront wave functions and cannot be directly determined from Euclidean lattice QCD. PDFs are currently determined from global analyses of a wide range of scattering data (see, for example, [4-10] for a selection of recent analyses).

Lattice QCD calculations have instead focused on the first few Mellin moments of PDFs, which can be related to matrix elements of local twist-two operators, where twist is the dimension minus the spin of the operator. The lattice regulator breaks rotational 
symmetry, which induces mixing between lattice operators that would not mix in the continuum. The mixing between twist-two operators of different mass dimension introduces power-divergent mixing on the lattice, preventing the extraction of more than three moments of PDFs $[11,12]$.

Recently a new approach to determining PDFs on the lattice was proposed, via Euclidean counterparts of PDFs generally referred to as quasi PDFs [13-19]. A similar framework was proposed in [20]. The quasi PDFs are Euclidean matrix elements determined at finite nucleon momentum. At large Euclidean momentum, the quasi PDFs can be related to the true PDFs through an effective theory expansion, the Large Momentum Effective Theory (LaMET).

Preliminary lattice calculations have been encouraging [15, 18], although both calculations have incorporated only a single lattice spacing and a full understanding of systematic uncertainties is far from complete. In particular, there are three challenges for the approach as it stands: the restriction to low nucleon momentum with the computational resources currently available; a full understanding of the renormalisation of extended Euclidean operators; and the precise relation between light-front PDFs and Euclidean quasi PDFs.

These difficulties can be broadly classified as either chiefly practical, or chiefly theoretical. The first challenge, that associated with the systematic uncertainties corresponding to low values of nucleon momentum on current lattices, is largely a practical issue. Studies in the spectator di-quark model [16] suggest that moderate improvements in computational resources, and new algorithms tailored to nucleons with large momentum [21], will likely solve this difficulty, at least to a precision that can contribute to global analyses of the PDFs in regions of parameter space that are experimentally inaccessible. We will not consider these practical difficulties any further and focus instead on the theoretical aspects of quasi PDFs.

We address one of the theoretical challenges by proposing a new approach to calculating quasi PDFs on the lattice, in which the lattice degrees of freedom are smeared via the gradient flow [22-24]. Using ringed fermions, which do not require any multiplicative wavefunction renormalization $[25,26]$, the corresponding lattice matrix elements remain finite in the continuum limit. This approach evades the problem of the power-divergence associated with the Wilson line operator that defines the quasi PDF. The renormalization of quasi PDFs has been viewed through the lens of heavy quark effective theory [17] and, more recently, a counterterm procedure has been proposed to remove this power-divergence [27, 28], but neither approach has been established beyond two loops in perturbation theory.

Here we examine the relation between the smeared quasi PDF and the light-front PDF, and focus on the limit in which the flow time is small compared to the length scale set by the nucleon momentum and the nucleon momentum is sufficiently large that higher twist effects can be neglected. In this limit, we express the moments of the smeared quasi PDF in terms of moments of the light-front PDF via a small flow-time expansion. The primary advantage of our approach is the finite continuum limit for nonperturbative matrix elements determined using lattice QCD. The matching between the smeared quasi PDF, regulated by the flow time, and the light-front PDF in, say, the $\overline{M S}$ scheme can be carried out in the continuum and is independent of the details of the nonperturbative lattice calculation. 
We start by revisiting the definitions of the light-front and quasi PDFs in section 2 . We then analyze the relation between the light-front and quasi PDFs in section 3 and derive an evolution equation for the matching kernel in section 4 . We present our summary and conclusions in section 5 .

\section{Distribution functions}

Throughout this work we focus on flavor non-singlet unpolarized quasi and light-front PDFs. The extension to polarized quasi PDFs is straightforward. The flavor singlet case introduces additional mixing with the gluon distribution, but the principles are similar. We also assume that the quarks are massless and ignore complications arising from the correct treatment of heavy flavors, a subject of continued study for light-front PDFs (for reviews, see, for example, [29-31]).

\subsection{Bare PDFs}

In the following section, when we use the term "bare" we mean finite matrix elements determined with some regulator at finite cutoff. We leave the regulator implicit in this discussion, although one can have in mind dimensional regularization if desired. These bare matrix elements require renormalization in some scheme before one can remove the regulator (or, on the lattice, take the continuum limit). This usage follows that of the extensive discussions of light-front PDFs in, for example, [3, 32].

We denote bare light-front PDFs by $f^{(0)}(\xi)$. Light-front PDFs are frequently represented by $f_{j / N}^{(0)}(\xi)$, where $j$ denotes the quark flavor and $N$ the nucleon species, but here we will be considering only non-singlet distributions, for which we can neglect mixing between parton species, and work with sufficient generality that the nucleon species is not relevant to our discussion. We use light-front coordinates, $\left(x^{+}, x^{-}, \boldsymbol{x}_{\mathrm{T}}\right)$ such that $x^{ \pm}=(t \pm z) / \sqrt{2}$, and define $\xi=k^{+} / P^{+}$. We use $\xi$ to distinguish this variable from the Bjorken- $x$ parameter that characterizes the kinematics of scattering experiments and is given in terms of the experimental momentum transfer $Q^{2}=-q^{2}$ and hadron momentum $P$ by $x=Q^{2} /(2 P \cdot q)$. The bare PDF is defined as [3]

$$
f^{(0)}(\xi)=\int_{-\infty}^{\infty} \frac{\mathrm{d} \omega^{-}}{4 \pi} e^{-i \xi P^{+} \omega^{-}}\left\langle P\left|T \bar{\psi}\left(0, \omega^{-}, \mathbf{0}_{\mathrm{T}}\right) W\left(\omega^{-}, 0\right) \gamma^{+} \frac{\lambda^{a}}{2} \psi(0)\right| P\right\rangle_{\mathrm{C}} .
$$

Here $T$ is the time-ordering operator, $\psi$ is a quark field, and the subscript $\mathrm{C}$ indicates that the vacuum expectation value has been subtracted (in other words, only connected contributions are included). The operator $W\left(\omega^{-}, 0\right)$ is the Wilson line,

$$
W\left(\omega^{-}, 0\right)=\mathcal{P} \exp \left[-i g_{0} \int_{0}^{\omega^{-}} \mathrm{d} y^{-} A_{\alpha}^{+}\left(0, y^{-}, \mathbf{0}_{\mathrm{T}}\right) T_{\alpha}\right],
$$

with $\mathcal{P}$ the path-ordering operator, $g_{0}$ the QCD bare coupling, and $A^{\mu}=A_{\alpha}^{\mu} T_{\alpha}$ the $\mathrm{SU}(3)$ gauge potential with generator $T_{\alpha}$ (summation over color index $\alpha$ is implicit). The target state, $|P\rangle$, is a spin-averaged, exact momentum eigenstate with relativistic normalization

$$
\left\langle P^{\prime} \mid P\right\rangle=(2 \pi)^{3} 2 P^{+} \delta\left(P^{+}-P^{\prime+}\right) \delta^{(2)}\left(\mathbf{P}_{\mathrm{T}}-\mathbf{P}_{\mathrm{T}}^{\prime}\right) .
$$


We define the moments of bare PDFs as

$$
a_{0}^{(n)}=\int_{0}^{1} \mathrm{~d} \xi \xi^{n-1}\left[f^{(0)}(\xi)+(-1)^{n} \bar{f}^{(0)}(\xi)\right]=\int_{-1}^{1} \mathrm{~d} \xi \xi^{n-1} f(\xi),
$$

where $\bar{f}^{(0)}(\xi)$ is the anti-quark PDF and the second equality follows from the relation of the quark to anti-quark PDFs

$$
f^{(0)}(-\xi)=-\bar{f}^{(0)}(\xi)
$$

which holds for the bare distributions if the quark and anti-quarks fields are classical, or quantized using light-front quantization [32].

We can relate these bare moments, $a_{0}^{(n)}$, to matrix elements of twist-two operators via

$$
\left\langle P\left|\mathcal{O}_{0}^{\left\{\mu_{1} \ldots \mu_{n}\right\}}\right| P\right\rangle=2 a_{0}^{(n)}\left(P^{\mu_{1}} \ldots P^{\mu_{n}}-\text { traces }\right) .
$$

Here the bare twist-two operators are

$$
\mathcal{O}_{0}^{\left\{\mu_{1} \cdots \mu_{n}\right\}}=i^{n-1} \bar{\psi}(0) \gamma^{\left\{\mu_{1}\right.} D^{\mu_{2}} \cdots D^{\left.\mu_{n}\right\}} \frac{\lambda^{a}}{2} \psi(0)-\text { traces }
$$

In these expressions the braces denote symmetrization, $D^{\mu}$ is the symmetric covariant derivative, $\lambda^{a}$ are $\mathrm{SU}(2)$ flavor matrices, and the subtraction of the trace terms ensures that the operator transforms irreducibly under $\mathrm{SU}(2)_{\mathrm{L}} \otimes \mathrm{SU}(2)_{\mathrm{R}}$.

\subsection{Renormalized PDFs}

To this point we have considered the bare light-front PDFs, with the understanding that such objects are evaluated with some regulator that renders the bare distributions finite. We now introduce renormalized light-front PDFs. We stress that in this section we consider a renormalization scheme that respects rotational symmetry and, for definiteness, one can have in mind the $\overline{M S}$ scheme. Complications will arise if a regulator that breaks rotational invariance, such as the lattice regulator, is used. We do not discuss such complications here, because we will avoid explicit computations of moments at finite lattice spacing. All correlation functions computed on the lattice can be renormalized and extrapolated to the continuum limit, provided that no power divergent mixing exists. In the next section, we propose a smeared correlation function that does not have power-divergent mixing.

In general, renormalized light-front PDFs are written in terms of a kernel, $\mathcal{Z}(\zeta / \xi, \mu)$, as

$$
f(\xi, \mu)=\int_{\xi}^{1} \frac{\mathrm{d} \zeta}{\zeta} \mathcal{Z}\left(\frac{\zeta}{\xi}, \mu\right) f^{(0)}(\zeta)
$$

where $\mu$ is some renormalization scale. We do not need to consider mixing between parton species for non-singlet distributions. In terms of the renormalized light-front PDF, the renormalized Mellin moments are

$$
a^{(n)}(\mu)=\int_{0}^{1} \mathrm{~d} \xi \xi^{n-1}\left[f(\xi, \mu)+(-1)^{n} \bar{f}(\xi, \mu)\right]=\int_{-1}^{1} \mathrm{~d} \xi \xi^{n-1} f(\xi, \mu),
$$


which can be related to matrix elements of renormalized twist-two operators, $\mathcal{O}^{\left\{\nu_{1} \ldots \nu_{n}\right\}}(\mu)=$ $Z_{\mathcal{O}}(\mu) \mathcal{O}_{0}^{\left\{\nu_{1} \ldots \nu_{n}\right\}}$, via

$$
\left\langle P\left|\mathcal{O}^{\left\{\nu_{1} \ldots \nu_{n}\right\}}(\mu)\right| P\right\rangle=2 a^{(n)}(\mu)\left(P^{\nu_{1}} \cdots P^{\nu_{n}}-\text { traces }\right) .
$$

This relation holds provided the light-front PDFs and twist-two operators are renormalized in the same scheme [32].

\subsection{Smeared quasi PDFs}

We construct a finite quasi PDF matrix element by smearing both the fermion and gauge fields via the gradient flow [22-24]. The gradient flow is a deterministic evolution of the original quark and gluon fields in a new dimension, generally referred to as the "flow time", towards a classical minimum of the QCD action [23, 24]. The flow-time evolution is chosen to remove ultraviolet fluctuations, which corresponds to smearing out the quark and gluon fields in real space, with a smearing scale that is proportional to the square-root of the flow time. Here we will not describe in detail the gradient flow, but refer the reader to the recent reviews [33, 34] for more details and applications.

For our purposes, it is sufficient that the gradient flow has the following properties. First, the gradient flow serves as a gauge-invariant ultraviolet regulator. Second, given a renormalized theory at zero flow time, the matrix elements of smeared fields are automatically finite, up to a multiplicative wave-function renormalization for the fermion fields [24], which can be removed by introducing ringed fermion fields [25, 26]. Third, the lattice matrix elements of smeared fields remain finite in the continuum limit, provided the flow time is fixed in physical units $[24,35]$. In essence, the gradient flow allows one to replace the lattice regulator with a new smearing-scale regulator. This last fact allows one to determine the continuum limit of lattice matrix elements of, for example, twist-two operators, without power-divergent mixing. In the continuum, because the gradient flow respects rotational symmetry, the mixing between twist-two operators is then reduced to ordinary mixing with coefficients that depend on the smearing scale and not powers of the inverse lattice spacing [35].

We denote the ringed fermion fields at flow time $\tau$ by $\bar{\chi}(x ; \tau)$ and $\chi(x ; \tau)$, and the corresponding Wilson line at the same flow time, constructed from the smeared gauge fields $B_{\mu}(x ; \tau)$, by $\mathcal{W}(0, z ; \tau)$. We start with the matrix element

$$
h^{(s)}\left(\frac{z}{\sqrt{\tau}}, \sqrt{\tau} P_{z}, \sqrt{\tau} \Lambda_{\mathrm{QCD}}, \sqrt{\tau} M_{\mathrm{N}}\right)=\frac{1}{2 P_{z}}\left\langle P_{z}\left|\bar{\chi}(z ; \tau) \mathcal{W}(0, z ; \tau) \gamma_{z} \frac{\lambda^{a}}{2} \chi(0 ; \tau)\right| P_{z}\right\rangle_{\mathrm{C}},
$$

which, being dimensionless, depends only on dimensionless combinations of scales. We note that the flow time has units of length-squared. The subscript $\mathrm{C}$ indicates that disconnected contributions to this matrix element have been removed. The ringed fermion fields require no wave function renormalization and this smeared matrix element is finite provided the flow time, $\tau$, is non-zero and fixed in physical units, because correlation functions constructed from smeared fields are finite [23, 24]. Note that divergences will appear in the limit of vanishing flow time and the matrix element will then require renormalization. 
We then define the quasi PDF $[13,14]$ as

$$
q^{(s)}\left(\xi, \sqrt{\tau} P_{z}, \sqrt{\tau} \Lambda_{\mathrm{QCD}}, \sqrt{\tau} M_{\mathrm{N}}\right)=\int_{-\infty}^{\infty} \frac{\mathrm{d} z}{2 \pi} e^{i \xi z P_{z}} P_{z} h^{(s)}\left(\sqrt{\tau} z, \sqrt{\tau} P_{z}, \sqrt{\tau} \Lambda_{\mathrm{QCD}}, \sqrt{\tau} M_{\mathrm{N}}\right),
$$

where $\xi$ is a dimensionless parameter that can be naively interpreted as the longitudinal momentum fraction of the parton in the nucleon $N$. This interpretation is not correct in Euclidean space, however, and instead $\xi$ should be viewed as a dimensionless momentum variable in a Fourier transformation.

In practice, the smeared matrix element $h$ is determined from lattice computations at finite lattice spacing, $a$, as

$$
h^{(s)}\left(\frac{z}{\sqrt{\tau}}, \sqrt{\tau} P_{z}, \sqrt{\tau} \Lambda_{\mathrm{QCD}}, \sqrt{\tau} M_{\mathrm{N}}\right)=\lim _{a \rightarrow 0} h\left(\frac{z}{a}, \frac{\sqrt{\tau}}{a}, a P_{z}, a \Lambda_{\mathrm{QCD}}, a M_{\mathrm{N}}\right),
$$

where $\sqrt{\tau} / a$ and $a P_{z}$ are held fixed and

$$
\begin{aligned}
& h\left(\frac{z}{a}, \frac{\sqrt{\tau}}{a}, a P_{z}, a \Lambda_{\mathrm{QCD}}, a M_{\mathrm{N}}\right)= \\
& \frac{1}{2 a P_{z}}\left\langle a P_{z}\left|\bar{\chi}\left(\frac{z}{a} ; \frac{\sqrt{\tau}}{a}\right) W\left(0, \frac{z}{a} ; \frac{\sqrt{\tau}}{a}\right) \gamma_{z} \frac{\lambda^{a}}{2} \chi\left(0 ; \frac{\sqrt{\tau}}{a}\right)\right| a P_{z}\right\rangle_{\mathrm{C}} .
\end{aligned}
$$

\section{Relation to light-front distributions}

We discuss the relation between quasi and light-front PDFs by examining the Mellin moments of these distributions, and using the connection between Mellin moments and matrix elements of local operators, which are twist-two in the case of light-front PDFs [36]. For the quasi PDFs, the local operators corresponding to the Mellin moments do not have a well-defined twist, but can be related to twist-two operators after subtracting higher twist effects and applying target-mass corrections [15, 18]. Although we consider smeared matrix elements in this work, the arguments regarding higher twist and target mass effects in $[15,18]$ still apply, because the flow time serves as an alternative gauge-invariant regulator to the lattice spacing.

We connect the Mellin moments of the quasi PDF to matrix elements of local operators in the following way. Working in axial gauge, $B_{z}(x ; \tau)=0$, the matrix element $h^{(s)}$ is

$$
\left.h^{(s)}\left(\frac{z}{\sqrt{\tau}}, \sqrt{\tau} P_{z}, \sqrt{\tau} \Lambda_{\mathrm{QCD}}, \sqrt{\tau} M_{\mathrm{N}}\right)\right|_{B_{z}=0}=\frac{1}{2 P_{z}}\left\langle P_{z}\left|\bar{\chi}(z ; \tau) \gamma_{z} \frac{\lambda^{a}}{2} \chi(0 ; \tau)\right| P_{z}\right\rangle_{\mathrm{C}} .
$$

We now substitute this expression into the definition of the quasi PDF, Equation (2.12), and integrate the resulting expression over the full range of $\xi$. In contrast to the light-front $\mathrm{PDF}$, this range extends from negative to positive infinity, giving

$$
\left.\int_{-\infty}^{\infty} \mathrm{d} \xi q^{(s)}\left(\xi, \sqrt{\tau} P_{z}, \sqrt{\tau} \Lambda_{\mathrm{QCD}}, \sqrt{\tau} M_{\mathrm{N}}\right)\right|_{B_{z}=0}=\left.h^{(s)}\left(0, \sqrt{\tau} P_{z}, \sqrt{\tau} \Lambda_{\mathrm{QCD}}, \sqrt{\tau} M_{\mathrm{N}}\right)\right|_{B_{z}=0} .
$$


Here we have used the relation $\delta\left(z P_{z}\right)=\delta(z) / P_{z}$, for $P_{z}>0$. We see that the first Mellin moment of the quasi PDF can be expressed in terms of the Euclidean matrix element of a local (smeared) operator.

We extend this argument to arbitrary moments of quasi PDFs by considering derivatives of the quasi distribution with respect to the spatial separation $z$ [3]. Inverting the Fourier transform in Equation (2.12), we have

$$
h^{(s)}\left(\frac{z}{\sqrt{\tau}}, \sqrt{\tau} P_{z}, \sqrt{\tau} \Lambda_{\mathrm{QCD}}, \sqrt{\tau} M_{\mathrm{N}}\right)=\int_{-\infty}^{\infty} \mathrm{d} \xi e^{-i \xi z P_{z}} q^{(s)}\left(\xi, \sqrt{\tau} P_{z}, \sqrt{\tau} \Lambda_{\mathrm{QCD}}, \sqrt{\tau} M_{\mathrm{N}}\right) .
$$

Applying derivatives with respect to the displacement $z$, we obtain

$$
\begin{aligned}
\left(\frac{i}{P_{z}} \frac{\partial}{\partial z}\right)^{n-1} h^{(s)} & \left(\frac{z}{\sqrt{\tau}}, \sqrt{\tau} P_{z}, \sqrt{\tau} \Lambda_{\mathrm{QCD}}, \sqrt{\tau} M_{\mathrm{N}}\right)= \\
& \int_{-\infty}^{\infty} \mathrm{d} \xi \xi^{n-1} e^{-i \xi z P_{z}} q^{(s)}\left(\xi, \sqrt{\tau} P_{z}, \sqrt{\tau} \Lambda_{\mathrm{QCD}}, \sqrt{\tau} M_{\mathrm{N}}\right)
\end{aligned}
$$

Defining the moments of the smeared quasi PDF, $b_{n}^{(s)}$, as

$$
b_{n}^{(s)}\left(\sqrt{\tau} P_{z}, \frac{\Lambda_{\mathrm{QCD}}}{P_{z}}, \frac{M_{\mathrm{N}}}{P_{z}}\right)=\int_{-\infty}^{\infty} \mathrm{d} \xi \xi^{n-1} q^{(s)}\left(\xi, \sqrt{\tau} P_{z}, \sqrt{\tau} \Lambda_{\mathrm{QCD}}, \sqrt{\tau} M_{\mathrm{N}}\right),
$$

and substituting the definition of the matrix element $h^{(s)}$, given in Equation (2.11), into Equation (3.4), in the limit that $z \rightarrow 0$ we obtain

$$
\begin{aligned}
b_{n}^{(s)}\left(\sqrt{\tau} P_{z}, \frac{\Lambda_{\mathrm{QCD}}}{P_{z}}, \frac{M_{\mathrm{N}}}{P_{z}}\right)_{B_{z}=0}= & \frac{c_{n}^{(s)}\left(\sqrt{\tau} P_{z}\right)}{2 P_{z}^{n}} \\
& \times\left\langle P_{z}\left|\left[\bar{\chi}(z ; \tau) \gamma_{z}\left(i \overleftarrow{\partial}_{z}^{n-1}\right) \frac{\lambda^{a}}{2} \chi(0 ; \tau)\right]_{z=0}\right| P_{z}\right\rangle_{\mathrm{C}}
\end{aligned}
$$

The perturbative coefficients, $c_{n}^{(s)}\left(\sqrt{\tau} P_{z}\right)$, capture potential singularities in the righthand side of Equation (3.4) in the limit of vanishing separation $z$ and vanishing flow time $\tau$, and follow from a smeared operator product expansion [35] approach to the nonlocal matrix element, as outlined in [15].

We restore gauge invariance to obtain our final expression for the moments of quasi PDFs in terms of Euclidean matrix elements of local operators:

$$
b_{n}^{(s)}\left(\sqrt{\tau} P_{z}, \frac{\Lambda_{\mathrm{QCD}}}{P_{z}}, \frac{M_{\mathrm{N}}}{P_{z}}\right)=\frac{c_{n}^{(s)}\left(\sqrt{\tau} P_{z}\right)}{2 P_{z}^{n}}\left\langle P_{z}\left|\left[\bar{\chi}(z ; \tau) \gamma_{z}\left(i \overleftarrow{D}_{z}\right)^{(n-1)} \frac{\lambda^{a}}{2} \chi(0 ; \tau)\right]_{z=0}\right| P_{z}\right\rangle_{\mathrm{C}}
$$

The local operators that appear in the matrix element on the right hand side of this expression are not twist-two operators: they are not symmetric and traceless. The discrepancy between these matrix elements and matrix elements of twist-two operators are given by corrections that appear in powers of $\Lambda_{\mathrm{QCD}}^{2} / P_{z}^{2}$ and $M_{\mathrm{N}}^{2} / P_{z}^{2}[15,18]$. The terms that scale as $\mathcal{O}\left(M_{\mathrm{N}}^{2} / P_{z}^{2}\right)$ correspond to target mass corrections [37, 38]. Although the appropriate interpretation of PDFs in the presence of these target mass corrections is 
subtle $[39,40]$, for our purposes it is sufficient that these non-leading corrections can be absorbed by writing $[15,18]$

$$
\begin{aligned}
b_{n}^{(s)}\left(\sqrt{\tau} P_{z}, \frac{\Lambda_{\mathrm{QCD}}}{P_{z}}\right)= & \frac{c_{n}^{(s)}\left(\sqrt{\tau} P_{z}\right)}{2 P_{z}^{n}}\left\langle P_{z}\left|\left[\bar{\chi}(z ; \tau) \gamma_{z}\left(i \overleftarrow{D}_{z}\right)^{(n-1)} \frac{\lambda^{a}}{2} \chi(0 ; \tau)\right]_{z=0}\right| P_{z}\right\rangle_{\mathrm{C}} \\
& \times K_{n}^{-1}\left(\frac{M_{\mathrm{N}}^{2}}{4 P_{z}^{2}}\right)
\end{aligned}
$$

where

$$
K_{n}\left(\frac{M_{\mathrm{N}}^{2}}{4 P_{z}^{2}}\right)=\sum_{j=0}^{n / 2}\left(\begin{array}{c}
n-j \\
j
\end{array}\right)\left(\frac{M_{\mathrm{N}}^{2}}{4 P_{z}^{2}}\right)^{j} .
$$

The corrected matrix elements on the right hand side of this equation can now be expanded in a Taylor series with respect to $\Lambda_{\mathrm{QCD}}^{2} / P_{z}^{2}$. The coefficients in this expansion represent higher twist effects that arise because the original matrix element is not a matrix element of a twist-two operator. The leading coefficient in this expansion is a twist-two contribution that can depend only on the nucleon structure and the flow time:

$$
b_{n}^{(s)}\left(\sqrt{\tau} P_{z}, \sqrt{\tau} \Lambda_{\mathrm{QCD}}\right)=c_{n}^{(s)}\left(\sqrt{\tau} P_{z}\right) b_{n}^{(s, \text { twist }-2)}\left(\sqrt{\tau} \Lambda_{\mathrm{QCD}}\right)+\mathcal{O}\left(\frac{\Lambda_{\mathrm{QCD}}^{2}}{P_{z}^{2}}\right),
$$

where, for $\Lambda_{\mathrm{QCD}}^{2} / P_{z}^{2} \ll 1$, the higher twist corrections can be ignored.

In summary, we assume that: first, we can correct exactly for target mass corrections; and second, we can take the momentum $P_{z}$ sufficiently large that higher twist effects are negligible. Then, under these assumptions, the moments of the smeared quasi PDFs are dimensionless products of perturbative coefficients and pure twist-two matrix elements, which are only functions of the dimensionless quantity $\sqrt{\tau} \Lambda_{\mathrm{QCD}}$, that contain information about the structure of the hadron.

\subsection{Short-distance expansion}

We can now relate the moments of the smeared quasi PDF $b_{n}^{(s, \text { twist }-2)}\left(\sqrt{\tau} \Lambda_{\mathrm{QCD}}\right)$, which are local matrix elements of smeared fields, to the renormalized moments of the lightfront PDFs, by using the properties of the gradient flow that arise from a short distance expansion $[23,25,26,41,42]$. The exponentially local nature of the smearing procedure allows for a short distance expansion of the smeared local operators in terms of renormalized operators in some renormalization scheme, such as the $\overline{M S}$ scheme. It is straightforward to show that this short distance expansion leads to

$$
b_{n}^{(s, \text { twist }-2)}\left(\sqrt{\tau} \Lambda_{\mathrm{QCD}}\right)=\widetilde{C}_{n}^{(0)}(\sqrt{\tau} \mu) a^{(n)}(\mu)+\mathcal{O}\left(\sqrt{\tau} \Lambda_{\mathrm{QCD}}\right),
$$

where $\mu$ is a renormalization scale. The leading order term in this expansion, $a^{(n)}(\mu)$, is the matrix element of a renormalized twist-two operator with the same gamma matrix and derivative structure as the smeared operator that appears in the matrix element on the left hand side. The higher order terms arise from higher dimension operators that enter the short distance expansion of the smeared matrix element. 
We now combine this short-distance expansion with Equation (3.10) to write

$$
b_{n}^{(s)}\left(\sqrt{\tau} \Lambda_{\mathrm{QCD}}\right)=C_{n}^{(0)}\left(\sqrt{\tau} \mu, \sqrt{\tau} P_{z}\right) a^{(n)}(\mu)+\mathcal{O}\left(\sqrt{\tau} \Lambda_{\mathrm{QCD}}, \frac{\Lambda_{\mathrm{QCD}}^{2}}{P_{z}^{2}}\right) .
$$

Both the leading short distance coefficient function, $C_{n}^{(0)}\left(\sqrt{\tau} \mu, \sqrt{\tau} P_{z}\right)$, and the higher order corrections can be computed in perturbation theory, so that this approximation can be systematically improved.

For the rest of this discussion, we will assume that we work in a regime in which there is a hierarchy of scales given by

$$
\Lambda_{\mathrm{QCD}}, M_{N} \ll P_{z} \ll \tau^{-1 / 2},
$$

so that power corrections and higher-twist effects can be ignored. We also assume that target mass corrections have been applied.

To relate the smeared quasi PDF with the light-front PDF, we introduce a kernel function, $Z\left(x, \sqrt{\tau} \mu, \sqrt{\tau} P_{z}\right)$, whose Mellin moments are given by

$$
\left[C_{n}^{(0)}\left(\sqrt{\tau} \mu, \sqrt{\tau} P_{z}\right)\right]^{-1}=\int_{-\infty}^{\infty} d x x^{n-1} Z\left(x, \sqrt{\tau} \mu, \sqrt{\tau} P_{z}\right)
$$

With this definition, and using the properties of multiplicative convolution, we find

$$
f(x, \mu)=\int_{-\infty}^{\infty} \frac{d \xi}{\xi} Z\left(\frac{x}{\xi}, \sqrt{\tau} \mu, \sqrt{\tau} P_{z}\right) q^{(s)}\left(\xi, \sqrt{\tau} \Lambda_{\mathrm{QCD}}\right)+\mathcal{O}\left(\sqrt{\tau} \Lambda_{\mathrm{QCD}}\right) .
$$

We introduce the inverse kernel through

$$
C_{n}^{(0)}\left(\sqrt{\tau} \mu, \sqrt{\tau} P_{z}\right)=\int_{-\infty}^{\infty} d x x^{n-1} \widetilde{Z}\left(x, \sqrt{\tau} \mu, \sqrt{\tau} P_{z}\right),
$$

which leads to

$$
q^{(s)}\left(x, \sqrt{\tau} \Lambda_{\mathrm{QCD}}, \sqrt{\tau} P_{z}\right)=\int_{-1}^{1} \frac{d \xi}{\xi} \widetilde{Z}\left(\frac{x}{\xi}, \sqrt{\tau} \mu, \sqrt{\tau} P_{z}\right) f(\xi, \mu)+\mathcal{O}\left(\sqrt{\tau} \Lambda_{\mathrm{QCD}}\right)
$$

Note that all of these relations are only valid if

$$
\Lambda_{Q C D}, M_{N} \ll P_{z} \ll \tau^{-1 / 2} .
$$

The kernel function can be computed in continuum perturbation theory, following the methods introduced in [23] and the examples in [17, 20, 27, 28]. Given that those computations are performed in the continuum, they are independent of the lattice formulation used to extract the smeared quasi-PDFs introduced in this paper and lattice computations of smeared quasi-PDFs can be performed with a variety of lattice actions, each of which results in the same universal continuum quasi-PDF. Consequently, lattice computations are disconnected from the matching procedure of these universal, continuum quasi-PDFs to the light-front PDFs. 
In both our approach, as in Ji's approach, the large nucleon momentum serves only to suppress higher twist contributions. We have, however, introduced a new scale, the (inverse) flow time, $\tau^{-1}$, that serves as a regulator of ultraviolet divergences that arise from external composite operators that define the matrix element. The scale $\tau^{-1}$ needs to be large relative to hadronic scales but remains finite. These requirements for the hierarchy of scales, expressed in Equation (3.18), are no different in nature than the requirements used to factor physical cross-sections into PDFs and Wilson coefficients and are similar in spirit to the factorization approach proposed in $[20,28]$. In this approach, the renormalization scale, which plays a similar role as our flow time scale, and the factorization scale are distinct and separate from the large momentum, which suppresses higher twist effects.

\section{DGLAP-like equation for the matching kernel}

Ignoring mixing between quark flavors and gluons (i.e. looking at the non-singlet distributions) the renormalized PDFs satisfy a DGLAP equation [43-45] that describes their scale dependence

$$
\mu \frac{\mathrm{d} f(x, \mu)}{\mathrm{d} \mu}=\frac{\alpha_{s}(\mu)}{\pi} \int_{x}^{1} \frac{\mathrm{d} y}{y} f(y, \mu) P\left(\frac{x}{y}\right) .
$$

Here $P(z)$ is a function whose moments are given by

$$
\int_{0}^{1} \mathrm{~d} x x^{n-1} P(x)=\gamma^{(n)}
$$

where

$$
\left[\mu \frac{\mathrm{d}}{\mathrm{d} \mu}-\frac{\alpha_{s}(\mu)}{\pi} \gamma^{(n)}\right] a^{(n)}(\mu)=0,
$$

and $\alpha_{s}(\mu)$ is the (renormalized) strong coupling constant.

Similarly, we can derive a DGLAP-like equation for the matching kernel that relates smeared quasi PDFs and light-front PDFs. We start from the small distance expansion in Equation (3.12), apply the renormalization group operator $\mu \mathrm{d} /(\mathrm{d} \mu)$, and use Equation (4.3) to derive a renormalization group equation for the short distance coefficient

$$
\left[\mu \frac{\mathrm{d}}{\mathrm{d} \mu}+\frac{\alpha_{s}(\mu)}{\pi} \gamma^{(n)}\right] C_{n}^{(0)}\left(\sqrt{\tau} \mu, \sqrt{\tau} P_{z}\right)=0+\mathcal{O}\left(\sqrt{\tau} \Lambda_{\mathrm{QCD}}\right),
$$

and its inverse

$$
\left[\mu \frac{\mathrm{d}}{\mathrm{d} \mu}-\frac{\alpha_{s}(\mu)}{\pi} \gamma^{(n)}\right]\left[C_{n}^{(0)}\left(\sqrt{\tau} \mu, \sqrt{\tau} P_{z}\right)\right]^{-1}=0+\mathcal{O}\left(\sqrt{\tau} \Lambda_{\mathrm{QCD}}\right) .
$$

We can obtain a DGLAP-like equation for the matching kernel by substituting Equations (3.14) and (4.2) into this renormalization group equation, to give

$$
\mu \frac{d}{d \mu} Z\left(x, \sqrt{\tau} \mu, \sqrt{\tau} P_{z}\right)=\frac{\alpha_{s}(\mu)}{\pi} \int_{x}^{\infty} \frac{d y}{y} Z\left(y, \sqrt{\tau} \mu, \sqrt{\tau} P_{z}\right) P\left(\frac{x}{y}\right),
$$

and

$$
\mu \frac{d}{d \mu} \widetilde{Z}\left(x, \sqrt{\tau} \mu, \sqrt{\tau} P_{z}\right)=-\frac{\alpha_{s}(\mu)}{\pi} \int_{x}^{\infty} \frac{d y}{y} \widetilde{Z}\left(y, \sqrt{\tau} \mu, \sqrt{\tau} P_{z}\right) P\left(\frac{x}{y}\right),
$$

up to corrections of $\mathcal{O}\left(\sqrt{\tau} \Lambda_{\mathrm{QCD}}\right)$. 


\section{Conclusion}

Parton distribution functions (PDFs) characterize nucleon structure in terms of the nucleon's constituent quarks and gluons. These PDFs are defined as matrix elements of light-front wave functions and cannot be directly calculated in Euclidean lattice QCD. In principle, however, the Mellin moments of PDFs can be calculated in lattice QCD, through matrix elements of twist-two operators. Unfortunately, these calculations are limited to the first few moments, because the hypercubic symmetry of the lattice regulator induces power-divergent mixing between twist-two operators of different mass dimension, obscuring the continuum limit of matrix elements determined on the lattice. Current determinations of PDFs rely on global analyses of data in a wide range of experimental channels and a determination of PDFs from first principles is lacking.

A new approach to determining PDFs in lattice QCD was recently proposed by Ji and subsequently, through a related framework, by Qiu and Ma. In this approach, one calculates Euclidean quasi PDFs at large nucleon momentum. Two recent lattice calculations provided promising results, but several aspects of the approach are yet to be fully understood. First, there is the practical issue of the systematic uncertainties associated with finite nucleon momenta in lattice calculations. This issue is likely to be resolved, to the extent that lattice calculations will have sufficient precision that results will provide useful input into global analyses where experimental data are inadequate, with improvements in computational resources and the algorithmic advances already underway. Second, there are theoretical issues to be clarified: the renormalization of the extended operator that defines the quasi PDFs; and the relation between the Euclidean quasi PDF and the light-front PDF, which to date had been analyzed through a factorization formula at one loop in perturbation theory.

We have addressed the first of these theoretical considerations by introducing a quasi PDF constructed from fields smeared via the gradient flow. We explicitly demonstrated that there is a simple relation between the Mellin moments of the smeared Euclidean quasi $\mathrm{PDF}$ and the renormalized Mellin moments of the light-front PDF, once nucleon mass corrections are incorporated and provided the flow time is small relative to the inverse nucleon momentum. Corrections to this relation appear at $\mathcal{O}\left(\Lambda_{\mathrm{QCD}}^{2} / P_{z}^{2}\right)$, where $P_{z}$ is the Euclidean momentum of the nucleon, and $\mathcal{O}\left(\sqrt{\tau} \Lambda_{\mathrm{QCD}}\right)$, where $\tau$ is the flow time. From this correspondence it follows that, provided $\Lambda_{Q C D}, M_{N} \ll P_{z} \ll \tau^{-1 / 2}$, the quasi PDF and light-front PDF can be matched through a convolution relation.

The chief advantage of our approach is that the gradient flow renders the quasi PDF finite in the continuum limit and evades the issues of the renormalization of the non-local operator that defines the quasi PDF on the lattice. The resulting continuum matrix elements are independent of the choices of discretized action used to undertake lattice QCD calculations and can be matched directly to the corresponding light-front PDFs in the $\overline{M S}$ scheme using continuum perturbation theory. Combined with a nonperturbative stepscaling procedure, this matching can be carried out at an energy sufficiently high that perturbative truncation errors are no longer uncontrolled. The nonperturbative implementation of our proposal is work in progress. 


\section{Acknowledgments}

We thank Xiangdong Ji, Herbert Neuberger, Jianwei Qiu, and Christian Weisz for enlightening discussions during the course of this work. We are particularly grateful to Carl Carlson for reading an early draft of this manuscript. K.O. has been supported by the U.S. Department of Energy through Grant Number DE- FG02-04ER41302, and through contract Number DE-AC05-06OR23177, under which JSA operates the Thomas Jefferson National Accelerator Facility.

Open Access. This article is distributed under the terms of the Creative Commons Attribution License (CC-BY 4.0), which permits any use, distribution and reproduction in any medium, provided the original author(s) and source are credited.

\section{References}

[1] CTEQ collaboration, R. Brock et al., Handbook of perturbative QCD: Version 1.0, Rev. Mod. Phys. 67 (1995) 157 [InSPIRE].

[2] G.F. Sterman, An introduction to quantum field theory, Cambridge University Press, (1994).

[3] J. Collins, Foundations of perturbative QCD, Cambridge University Press, (2011).

[4] F. Arbabifar, A.N. Khorramian and M. Soleymaninia, QCD analysis of polarized DIS and the SIDIS asymmetry world data and light sea-quark decomposition, Phys. Rev. D 89 (2014) 034006 [arXiv: 1311.1830] [INSPIRE].

[5] NNPDF collaboration, R.D. Ball, S. Forte, A. Guffanti, E.R. Nocera, G. Ridolfi and J. Rojo, Unbiased determination of polarized parton distributions and their uncertainties, Nucl. Phys. B $\mathbf{8 7 4}$ (2013) 36 [arXiv:1303.7236] [InSPIRE].

[6] P. Jimenez-Delgado, A. Accardi and W. Melnitchouk, Impact of hadronic and nuclear corrections on global analysis of spin-dependent parton distributions, Phys. Rev. D 89 (2014) 034025 [arXiv: 1310 .3734] [INSPIRE].

[7] Jefferson Lab Angular Momentum (JAM) collaboration, P. Jimenez-Delgado, H. Avakian and W. Melnitchouk, Constraints on spin-dependent parton distributions at large $x$ from global QCD analysis, Phys. Lett. B 738 (2014) 263 [arXiv:1403.3355] [INSPIRE].

[8] S. Dulat et al., New parton distribution functions from a global analysis of quantum chromodynamics, Phys. Rev. D 93 (2016) 033006 [arXiv: 1506.07443] [INSPIRE].

[9] T.-J. Hou et al., Reconstruction of Monte Carlo replicas from Hessian parton distributions, arXiv: 1607.06066 [INSPIRE].

[10] F. Taghavi-Shahri, H. Khanpour, S. Atashbar Tehrani and Z. Alizadeh Yazdi, Next-to-next-to-leading order QCD analysis of spin-dependent parton distribution functions and their uncertainties: Jacobi polynomials approach, Phys. Rev. D 93 (2016) 114024 [arXiv: 1603.03157] [INSPIRE].

[11] W. Detmold, W. Melnitchouk and A.W. Thomas, Parton distributions from lattice QCD, Eur. Phys. J. direct 3 (2001) 13 [hep-lat/0108002] [INSPIRE].

[12] W. Detmold, W. Melnitchouk and A.W. Thomas, Extraction of parton distributions from lattice QCD, Mod. Phys. Lett. A 18 (2003) 2681 [hep-lat/0310003] [INSPIRE]. 
[13] X. Ji, Parton Physics on a Euclidean Lattice, Phys. Rev. Lett. 110 (2013) 262002 [arXiv:1305.1539] [INSPIRE].

[14] X. Ji, Parton Physics from Large-Momentum Effective Field Theory, Sci. China Phys. Mech. Astron. 57 (2014) 1407 [arXiv: 1404.6680] [INSPIRE].

[15] H.-W. Lin, J.-W. Chen, S.D. Cohen and X. Ji, Flavor Structure of the Nucleon Sea from Lattice QCD, Phys. Rev. D 91 (2015) 054510 [arXiv:1402.1462] [INSPIRE].

[16] L. Gamberg, Z.-B. Kang, I. Vitev and H. Xing, Quasi-parton distribution functions: a study in the diquark spectator model, Phys. Lett. B 743 (2015) 112 [arXiv:1412.3401] [INSPIRE].

[17] X. Ji and J.-H. Zhang, Renormalization of quasiparton distribution, Phys. Rev. D 92 (2015) 034006 [arXiv: 1505.07699] [INSPIRE].

[18] C. Alexandrou et al., Lattice calculation of parton distributions, Phys. Rev. D 92 (2015) 014502 [arXiv: 1504.07455] [INSPIRE].

[19] J.-W. Chen, S.D. Cohen, X. Ji, H.-W. Lin and J.-H. Zhang, Nucleon Helicity and Transversity Parton Distributions from Lattice QCD, Nucl. Phys. B 911 (2016) 246 [arXiv: 1603.06664] [INSPIRE].

[20] Y.-Q. Ma and J.-W. Qiu, Extracting Parton Distribution Functions from Lattice QCD Calculations, arXiv:1404.6860 [INSPIRE].

[21] G.S. Bali, B. Lang, B.U. Musch and A. Schäfer, Novel quark smearing for hadrons with high momenta in lattice QCD, Phys. Rev. D 93 (2016) 094515 [arXiv: 1602.05525] [INSPIRE].

[22] R. Narayanan and H. Neuberger, Infinite $N$ phase transitions in continuum Wilson loop operators, JHEP 03 (2006) 064 [hep-th/0601210] [INSPIRE].

[23] M. Lüscher and P. Weisz, Perturbative analysis of the gradient flow in non-abelian gauge theories, JHEP 02 (2011) 051 [arXiv: 1101.0963] [INSPIRE].

[24] M. Lüscher, Chiral symmetry and the Yang-Mills gradient flow, JHEP 04 (2013) 123 [arXiv: 1302.5246] [INSPIRE].

[25] H. Makino and H. Suzuki, Lattice energy-momentum tensor from the Yang-Mills gradient flow-inclusion of fermion fields, PTEP 2014 (2014) 063B02 [Erratum ibid. 2015 (2015) 079202] [arXiv: 1403.4772] [INSPIRE].

[26] K. Hieda and H. Suzuki, Small flow-time representation of fermion bilinear operators, Mod. Phys. Lett. A 31 (2016) 1650214 [arXiv:1606. 04193] [INSPIRE].

[27] J.-W. Chen, X. Ji and J.-H. Zhang, Improved quasi parton distribution through Wilson line renormalization, Nucl. Phys. B 915 (2017) 1 [arXiv:1609.08102] [INSPIRE].

[28] T. Ishikawa, Y.-Q. Ma, J.-W. Qiu and S. Yoshida, Practical quasi parton distribution functions, arXiv: 1609.02018 [INSPIRE].

[29] R.S. Thorne and W.K. Tung, PQCD Formulations with Heavy Quark Masses and Global Analysis, arXiv:0809.0714 [INSPIRE].

[30] F. Olness and I. Schienbein, Heavy Quarks: Lessons Learned from HERA and Tevatron, Nucl. Phys. Proc. Suppl. 191 (2009) 44 [arXiv:0812.3371] [InSPIRE].

[31] SM and NLO Multileg Working Group collaboration, T. Binoth et al., The SM and NLO Multileg Working Group: Summary report, arXiv:1003.1241 [INSPIRE]. 
[32] J.C. Collins and D.E. Soper, Parton Distribution and Decay Functions, Nucl. Phys. B 194 (1982) 445 [INSPIRE].

[33] M. Lüscher, Future applications of the Yang-Mills gradient flow in lattice QCD, PoS (LATTICE 2013) 016 [arXiv: 1308.5598] [INSPIRE].

[34] A. Ramos, The Yang-Mills gradient flow and renormalization, PoS (LATTICE2014) 017 [arXiv: 1506. 00118] [INSPIRE].

[35] C. Monahan and K. Orginos, Locally smeared operator product expansions in scalar field theory, Phys. Rev. D 91 (2015) 074513 [arXiv:1501.05348] [InSPIRE].

[36] N.H. Christ, B. Hasslacher and A.H. Mueller, Light cone behavior of perturbation theory, Phys. Rev. D 6 (1972) 3543 [InSPIRE].

[37] O. Nachtmann, Positivity constraints for anomalous dimensions, Nucl. Phys. B 63 (1973) 237 [INSPIRE].

[38] H. Georgi and H.D. Politzer, Electroproduction scaling in an asymptotically free theory of strong interactions, Phys. Rev. D 9 (1974) 416 [INSPIRE].

[39] F.M. Steffens, M.D. Brown, W. Melnitchouk and S. Sanches, Parton distributions in the presence of target mass corrections, Phys. Rev. C 86 (2012) 065208 [arXiv:1210.4398] [INSPIRE].

[40] S. Taheri Monfared, Z. Haddadi and A.N. Khorramian, Target mass corrections and higher twist effects in polarized deep-inelastic scattering, Phys. Rev. D 89 (2014) 074052 [arXiv: 1405.4633] [INSPIRE].

[41] H. Suzuki, Energy-momentum tensor from the Yang-Mills gradient flow, PTEP 2013 (2013) $083 \mathrm{~B} 03$ [Erratum ibid. 2015 (2015) 079201] [arXiv: 1304.0533] [INSPIRE].

[42] FLowQCD collaboration, M. Asakawa, T. Hatsuda, E. Itou, M. Kitazawa and H. Suzuki, Thermodynamics of SU(3) gauge theory from gradient flow on the lattice, Phys. Rev. D 90 (2014) 011501 [arXiv: 1312.7492] [INSPIRE].

[43] V.N. Gribov and L.N. Lipatov, $e^{+} e^{-}$pair annihilation and deep inelastic e $p$ scattering in perturbation theory, Sov. J. Nucl. Phys. 15 (1972) 675 [INSPIRE].

[44] Y.L. Dokshitzer, Calculation of the Structure Functions for Deep Inelastic Scattering and $e^{+} e^{-}$Annihilation by Perturbation Theory in Quantum Chromodynamics., Sov. Phys. JETP 46 (1977) 641 [INSPIRE].

[45] G. Altarelli and G. Parisi, Asymptotic Freedom in Parton Language, Nucl. Phys. B 126 (1977) 298 [INSPIRE]. 